\title{
Visual control of posture in real and virtual environments
}

\author{
Jonathan W. Kelly AND BERnhaRd Riecke \\ Vanderbilt University, Nashville, Tennessee \\ AND \\ JACK M. LOOMIS AND ANDREW C. BEALL \\ University of California, Santa Barbara, California
}

\begin{abstract}
In two experiments, we investigated the stabilizing influence of vision on human upright posture in real and virtual environments. Visual stabilization was assessed by comparing eyes-open with eyes-closed conditions while subjects attempted to maintain balance in the presence of a stable visual scene. Visual stabilization in the virtual display was reduced, as compared with real-world viewing. This difference was partially accounted for by the reduced field of view in the virtual display. When the retinal flow in the virtual display was removed by using dynamic random-dot stereograms with single-frame lifetimes (cyclopean stimuli), vision did not stabilize posture. There was also an overall larger stabilizing influence of vision when more unstable stances were adopted (e.g., one-foot, as compared with side-by-side, stance). Reducing the graphics latency of the virtual display by $63 \%$ did not increase visual stabilization in the virtual display. Other visual and psychological differences between real and virtual environments are discussed.
\end{abstract}

Two lines of evidence underscore the importance of visual input in the control of posture (balance). First, occluding vision increases standing body sway by $200 \%-300 \%$ (Begbie, 1967; Diener, Dichgans, Bacher, \& Gompf, 1984; Edwards, 1946; Paulus, Straube, \& Brandt, 1984; Witkin \& Wapner, 1950). Experimental degradation of both visual acuity (by placing semitransparent plastic foils over the eyes) and field of view (FOV) has been shown to increase standing sway, relative to unobstructed vision (Paulus et al., 1984), and naturally occurring visual deficiencies are considered to be a major risk factor for falls in the elderly (Harwood, 2001). Second, the swinging room paradigm has been used to demonstrate that displacement of the visual environment produces a compensatory postural response (Lee \& Lishman, 1975). Presumably, the imposed visual motion is interpreted as self-motion, and a postural response is generated in an attempt to compensate for this perceived self-motion. Further solidifying this relationship between vision and posture is the finding that sinusoidal motion of the visual environment produces postural responses at the same frequency as the environmental motion (Bardy, Warren, \& Kay, 1996, 1999; Dijkstra, Gielen, \& Melis, 1992; Dijkstra, Schöner, \& Gielen, 1994; van Asten, Gielen, \& van der Gon, 1988a, 1988b).

Given the aforementioned results establishing an important role for vision in maintaining balance, the present experiments were designed with two primary goals in mind: (1) to determine whether the stabilizing influence of vision is comparable in real and virtual environments and, if not, to determine potential causes for differences, and (2) to assess the hypothesis that optic flow, a 2-D motion-based stimulus, is an important visual cue used to maintain balance.

\section{Postural Control in Virtual Reality}

Much of our understanding of the visual control of posture has come through experiments using simulated visual displays (Bardy et al., 1996, 1999; Bronstein \& Buckwell, 1997; Cunningham, Nusseck, Teufel, Wallraven, \& Bülthoff, 2006; Dijkstra et al., 1992, 1994; Kelly, Loomis, \& Beall, 2005; Mitra, 2003; Mitra \& Fraizer, 2004; van Asten et al., 1988a, 1988b). It is often assumed in these experiments that any variable affecting postural control in virtual reality (VR) would have a similar effect if implemented in a real environment (but for exceptions, see Cunningham et al., 2006; Stoffregen, Bardy, Merhi, \& Oullier, 2004). However, visual stimulation in virtual displays can differ in potentially important ways from real-world viewing. Some of these differences, relative to real-world viewing, include reduced FOV, graphics update latency in response to observer movement, fixed accommodative distance, optical distortion, display quantization, and the added weight when a head-mounted display (HMD) is worn. Recently, Stoffregen et al. (2004) compared postural responses to real and virtual swinging rooms and matched the FOV in the two different viewing environments. When the real display was viewed, the coupling between postural sway 
and room motion increased with increasing room motion amplitude. In contrast, no such effect of room amplitude was found with the virtual display, suggesting that postural control in virtual environments may not be representative of postural control in real environments. However, the differences in response to the two display types could be a result of differences in visual cues. When the real display was viewed, visual expansion of the scene, as well as binocular vision, provided redundant information about the room motion. In the virtual display, the visual expansion was well matched to that of the real world, but the binocular cues were in conflict with the visual expansion of the scene: As the virtual wall expanded and contracted (i.e., moved virtually toward and away from the subject), the binocular cues never changed and, thus, provided conflicting motion information that could have altered the subjects' postural responses.

Recognizing that the display quantization of the virtual scene could also be responsible for the observed differences, Stoffregen et al. (2004) manipulated the size of the texture elements in the two displays. They expected that larger texture elements, which are less subject to spatial aliasing, would produce a larger postural response to the virtual environment. Instead, they found no effect of texture element size, suggesting that display quantization was not a major influence on visual control of posture with their virtual display. ${ }^{1}$

Subsequently, Cunningham et al. (2006) used a large FOV virtual display $\left(220^{\circ}\right.$ horizontal $\times 50^{\circ}$ vertical $)$ to test postural responses to a swinging room while varying the room oscillation amplitude. In contrast to Stoffregen et al. (2004), they found a clear increase in body sway with increasing room oscillation amplitude. There are, however, multiple differences that could account for the discrepant results. First, Cunningham et al. used a wide range of room motion amplitudes, from 2.5 to $80 \mathrm{~cm}$, whereas Stoffregen et al. (2004) used amplitudes between 2 and $22 \mathrm{~cm}$. Second, Cunningham et al. used a frequencymodulated oscillatory room motion and a somewhat different method of data analysis. Third, Cunningham et al. displayed a naturalistic visual scene on a cylindrical projection screen that provided a larger FOV at an increased viewing distance $(3.5 \mathrm{~m}$, as compared with $0.8 \mathrm{~m}$ in the study by Stoffregen et al., 2004), which together may have helped to compensate for some of the other shortcomings of virtual displays. Overall, the results from Cunningham et al. were, in fact, consistent with previous real-world data. Although Cunningham et al. succeeded in demonstrating the influence of room amplitude on postural sway, they did not directly compare this with postural responses to real environments. To that end, Stoffregen et al. (2004) provide the only direct comparison of postural control in real and virtual environments.

Although the swinging room paradigm has been successful in advancing the understanding of postural control, other studies have employed stationary visual environments to explore the link between vision and posture. These experiments have been conducted using real environments (Brandt, Arnold, Bles, \& Kapteyn, 1980; Diener et al., 1984; Edwards, 1946; Guerraz, Sakellari, Burchill,
\& Bronstein, 2000; Lasley, Hamer, Dister, \& Cohn, 1991; Paulus et al., 1984; Paulus, Straube, Krafczyk, \& Brandt, 1989; Stoffregen, Smart, Bardy, \& Pagulayan, 1999; Witkin \& Wapner, 1950), as well as virtual environments (Dijkstra et al., 1992; Kelly et al., 2005; Mitra, 2003; Mitra \& Fraizer, 2004). To our knowledge, no direct comparison of postural control in stationary real and virtual environments has been conducted. However, a comparison of the results from separate studies provides some insights. For example, Paulus et al. (1989) measured body sway in the presence of a stationary real-world object, which was placed between 10 and $250 \mathrm{~cm}$ from the observer. Similarly, Dijkstra et al. (1992, Experiment 1) measured body sway when a virtual target was placed between 10 and $110 \mathrm{~cm}$ from the observer. Both studies converged on the same general finding: Natural body sway increases with increased distance to the visual target.

Although researchers frequently use VR to study postural control, little work has been done to validate this research tool (see, however, Cunningham et al., 2006; Stoffregen et al., 2004). To that end, the primary goal of these experiments was to provide a direct comparison of postural control in stationary real and virtual environments. In anticipation of potential differences between the two environment types, an additional viewing condition was included in which subjects viewed the real environment through an FOV limiter, which restricted FOV to levels comparable to those with the HMD. This was chosen as a likely source of potential differences on the basis of work by Paulus et al. (1984), in which FOV limiters decreased visual stabilization.

\section{Postural Control and Optic Flow}

One interpretation of the swinging room experiments, in which postural adjustments are made in response to a visually oscillating room, is that self-motion through the environment is perceived through optic flow, or the changing angular positions of points in the environment. Optic flow traditionally is defined with respect to the head. For a stationary environment, optic flow reflects translations and rotations of the head, but not rotations of the eye (Gibson, 1950; Nakayama \& Loomis, 1974). Optic flow is considered to be the distal stimulus, and retinal flow is the proximal stimulus to which the visual system responds. Retinal flow is a function of both the head-centric optic flow and rotations of the eye with respect to the head. In natural environments, relative motion between environment and observer induces retinal flow with motion energy. However, there are other types of motion stimuli that do not have motion energy and are referred to as second- and third-order motion (Lu \& Sperling, 1995, 1996). Some researchers have suggested that in order to maintain an upright stance, postural responses are produced to minimize the optic flow (Lee \& Lishman, 1975; Schöner, 1991; van Asten et al., 1988a; Warren, 1998; Warren, Kay, \& Yilmaz, 1996). Natural body sway in everyday environments produces expanding and contracting optic flow, and a simple way to maintain balance is to adjust posture in a direction opposite the optic flow pattern so as to nullify the optic flow.

The swinging room experiments by Stoffregen et al. (2004) provide evidence that optic flow is not the only 
visual cue used for postural control, although the authors themselves do not make this claim. In their experiments, optic flow cues were very well matched between the real and the virtual environments, but postural responses to the two environment types were quite different. van Asten et al. (1988a) measured postural responses to a virtual swinging room while observers fixated a stationary target in the center of the screen. They found that the phase of postural responses occasionally shifted by $180^{\circ}$ relative to the room oscillations, and the authors suggested that these phase shifts resulted from a changing interpretation of the scene motion. When observers interpreted the scene motion as self-motion through the environment, they made postural responses in phase with the room motion. When observers interpreted the scene motion as motion of the fixation target, they made postural responses out of phase with the room motion. This result implies that optic flow is, at most, only partially responsible for postural control and that a higher level interpretation of the scene can affect stability.

Work by Kelly et al. (2005) has argued that perceived relative motion between the 3-D environment and self can be used to control posture. By manipulating stereo depth cues, they created two situations with equivalent optic flow but different 3-D structures. Using these stimuli, they investigated the role of perceived environmentrelative motion in controlling posture. In one case, the 3-D scene appeared stationary, and in the other case, the scene appeared to move concomitantly with self-motion. Importantly, optic flow was the same for both stimuli. In the latter case, subjects were less stable, leading to the conclusion that perceived self-motion relative to the 3-D environment influences postural control, independently of optic flow. If optic flow were the only stimulus used to control posture, any changes in the 3-D structure of the scene should not have affected postural stability.

To further investigate the roles of retinal flow and perceived environment-relative motion, Experiment $1 \mathrm{em}-$ ployed a technique for creating interactive virtual environments with 3-D structure but no retinal flow. In ordinary environments, retinal flow and 3-D environment motion are in close correspondence. The subsequent experiment used a new method to produce a binocularly defined environment without retinal flow and, thereby, further investigate the role of perceived environment-relative motion in controlling posture. Other recent studies have shown that complex behaviors, such as catching a ball, avoiding obstacles, and judging heading, can be performed without retinal flow (Loomis, Beall, Macuga, Kelly, \& Smith, 2006; Macuga, Loomis, Beall, \& Kelly, 2006). Experiment 1 examined whether posture can be controlled without retinal flow in a stationary virtual environment.

\section{EXPERIMENT 1}

The two main goals of Experiment 1 were (1) to compare the role of vision in postural control in stationary real and virtual environments and (2) to investigate the role of retinal flow in postural control. In addition, Experiment 1 manipulated subject stance while the different visual dis- plays were viewed. Postural control researchers commonly attempt to reduce subject stability by having subjects stand on foam pads or assume difficult stances, with the expectation that reduced baseline stability will result in a greater influence of vision. The subjects in Experiment 1 assumed three different stances of varying stability during testing: side by side, heel to toe, and one foot. The stance manipulation was added to provide a more complete understanding of visually controlled posture. For example, visually controlled posture may be different in real and virtual environments when a stable stance is assumed, but these differences could be reduced if greater demands are placed on the visual system when a less stable stance is assumed. For all of the visual displays and stances, body sway was measured both with and without visual input, with the purpose of identifying cases in which the visual stimulus led to a reduction in body sway.

\section{Method}

Subjects. Eight undergraduate students ( 2 of them female) at the University of California, Santa Barbara, participated in exchange for course credit. All the subjects were verified to have at least 20-20 vision and $80 \%$ stereopsis, as measured on a Keystone orthoscope.

Design. Experiment 1 employed a $4 \times 3 \times 2$ full factorial design. There were four levels of visual display (real environment with full FOV, real environment with restricted FOV, virtual environment, and virtual environment with no retinal flow), three levels of stance (feet placed side by side, feet placed heel to toe, and one foot), and two levels of vision (vision or no vision). All variables were manipulated within subjects. Visual display type was blocked, and participation order in the four visual display conditions was counterbalanced using a balanced Latin square design. Within each block of visual display type, the order of stance was randomized. For each stance, the order of vision and no-vision trials was also randomized.

Stimuli and Materials. When viewing the real environment, the subjects viewed the outside of a closed lab door (see Figure 1). This was chosen due to the detailed texture of the door, along with the high contrast edges where the door contacted the wall. When viewing the real environment with reduced FOV, the subjects donned a purpose-built FOV limiter that reduced the visible binocular FOV to $50^{\circ}$ horizontal $\times 38^{\circ}$ vertical. This FOV was designed to equal

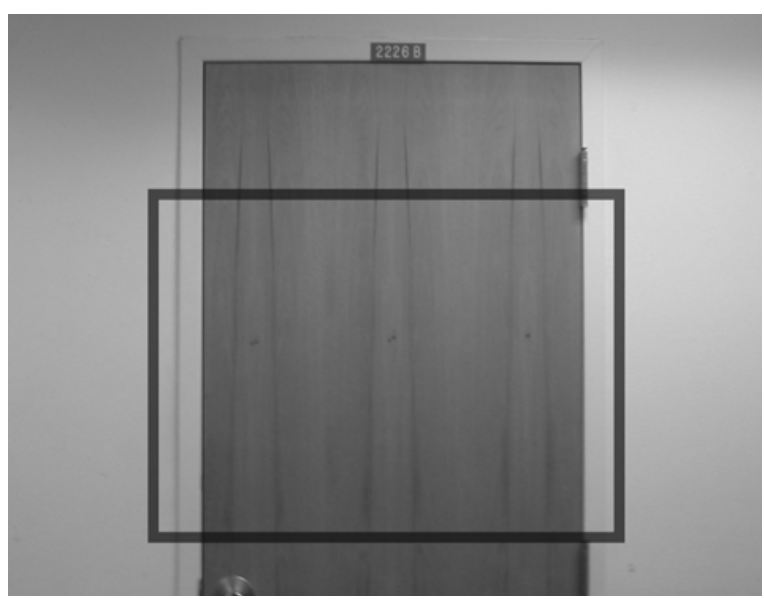

Figure 1. Photograph of the visual scene used in Experiments 1 and 2 . The gray box represents the visual scene when the field of view (FOV) was reduced by the FOV limiter or by the headmounted display. 
the limited FOV produced by the HMD used for displaying virtual environments. The gray outline in Figure 1 approximates the visible scene when the FOV limiter is worn. When viewing the textured virtual environment, the subjects viewed a photorealistic virtual world textured with photographs taken of the real environment. The textured virtual environment was visually similar to the real environment, except for limitations introduced by the VR system itself.

For the virtual display with no retinal flow, the environment was rendered using a cyclopean stimulus, where each graphics frame was a new random-dot stereogram. Each stereogram consisted of 1,000 points (each 1 pixel in size) randomly distributed across the FOV. On each new graphics frame, a new random-dot stereogram was drawn that reflected any changes in the scene geometry due to observer motion relative to the environment. The cyclopean stimulus contained no correlated motion from one frame to the next and, thus, no retinal flow information about the simulated environment or its movement resulting from observer motion. Conceptually speaking, 1,000 dots were cast onto the 3-D environment visible within the FOV. The appropriate screen coordinates were then calculated for each eye, taking interpupillary separation into account. This process was then repeated for each graphics frame. Because the cyclopean stimulus conveys information only through stereo cues, the same virtual environment could not be used for this condition. Instead, a new environment was created by placing several cones, which protruded from a frontoparallel wall. The cones were $20 \mathrm{~cm}$ long with a 10 -cm diameter at their base and were randomly scattered across the wall. Approximately $10-12$ of these cones were visible at any given time within the subject's FOV.

Virtual stimuli were presented using immersive VR experienced through an HMD (Virtual Research V8) with $640 \times 480$ resolution LCD panels refreshed at $60 \mathrm{~Hz}$. The FOV was $50^{\circ}$ horizontal $\times 38^{\circ}$ vertical. Projectively correct images were rendered by a $2.2-\mathrm{GHz}$ Pentium 4 processor with a GeForce 4 graphics card, using Vizard software (from WorldViz, Santa Barbara, CA). The subjects' head orientation was tracked with a 3-axis orientation sensor (IS300 from InterSense Inc., Bedford, MA), and head position was tracked three-dimensionally by a passive optical position sensing system (Precision Position Tracker, PT X4 from WorldViz). The end-to-end latency, or the delay between subject head motion and display update, was measured to be $90 \mathrm{msec}$, on average. ${ }^{2}$ Head position and orientation were recorded at $60 \mathrm{~Hz}$.

Procedure. For all visual displays and stances, the subjects removed their shoes and stood $1 \mathrm{~m}$ from the visual scene. For each trial, the subjects were instructed as to which stance they were to assume and whether their eyes would be opened or closed. The subjects were allowed to choose which foot was placed in front/behind for the heel-totoe stance, as well as which foot they stood on for the one-foot stance. However, they were required to use the same positioning throughout the experiment. In all cases, they were instructed to do their best to remain still. The trial began when the subject was in position and lasted for $60 \mathrm{sec}$. A bell indicated the beginning and end of each trial. The subjects took a short break (1-2 min) between blocks of visual display.

\section{Results}

The head position data for a typical subject in the realworld, unrestricted FOV condition are plotted in Figure 2. As in previous postural control studies (Kelly et al., 2005; Lasley et al., 1991; Okuzumi, Tanaka, \& Nakamura, 1996; Riley, Mitra, Stoffregen, \& Turvey, 1997; Stoffregen et al., 1999), the standard deviation of the subject's head position was calculated in both the lateral and the anterior-posterior (AP) body axes. To assess the stabilizing influence of vision, these standard deviations were combined to calculate a Romberg quotient for each condition - that is, the ratio of standard deviations with eyes closed and eyes open (Van Parys \& Njiokiktjien, 1976). A Romberg quotient greater than 1.0 indicates a stabilizing influence of vision.

Separate 4 (visual condition: real with full FOV, real with reduced FOV, VR, and cyclopean VR) $\times 3$ (stance: side by side, heel to toe, and one foot) repeated measures ANOVAs were conducted using the lateral and AP Romberg quotients. For lateral body sway (presented in Figure 3), there was a main effect of condition $[F(3,21)=6.81, p=.002$, $\left.\eta_{\mathrm{p}}^{2}=.49\right]$. Within-subjects contrasts showed that real-world unrestricted viewing produced marginally larger Romberg quotients than did real-world restricted FOV condition $\left[F(1,7)=4.33, p=.076, \eta_{\mathrm{p}}^{2}=.38\right]$. The real-world conditions, both with and without restricted FOV, produced larger Romberg quotients than did the $\operatorname{VR}[F(1,7)=7.27$, $p=.031, \eta_{\mathrm{p}}^{2}=.51$, and $F(1,7)=6.71, p=.036, \eta_{\mathrm{p}}^{2}=.49$, respectively] and the cyclopean $\operatorname{VR}[F(1,7)=7.82, p=$ $.027, \eta_{\mathrm{p}}^{2}=.53$, and $F(1,7)=6.85, p=.035, \eta_{\mathrm{p}}^{2}=.49$, respectively] conditions, which were not significantly different from one another $\left[F(1,7)=0.12\right.$, n.s., $\left.\eta_{\mathrm{p}}^{2}=.02\right]$. The main effect of stance was also significant $[F(2,14)=8.09$, $\left.p=.005, \eta_{\mathrm{p}}^{2}=.54\right]$, where Romberg quotients for the one-foot and heel-to-toe stances were significantly larger than those for the side-by-side stance $[F(1,7)=14.88, p=$ $.006, \eta_{\mathrm{p}}^{2}=.68$, and $F(1,7)=10.80, p=.013, \eta_{\mathrm{p}}^{2}=.61$, respectively]. There was no interaction between display and stance $\left[F(6,42)=1.90\right.$, n.s., $\left.\eta_{\mathrm{p}}^{2}=.21\right]$. One-sample $t$ tests were conducted to determine which conditions produced Romberg quotients greater than 1.0, indicating a stabilizing effect of vision. The results revealed that the side-by-

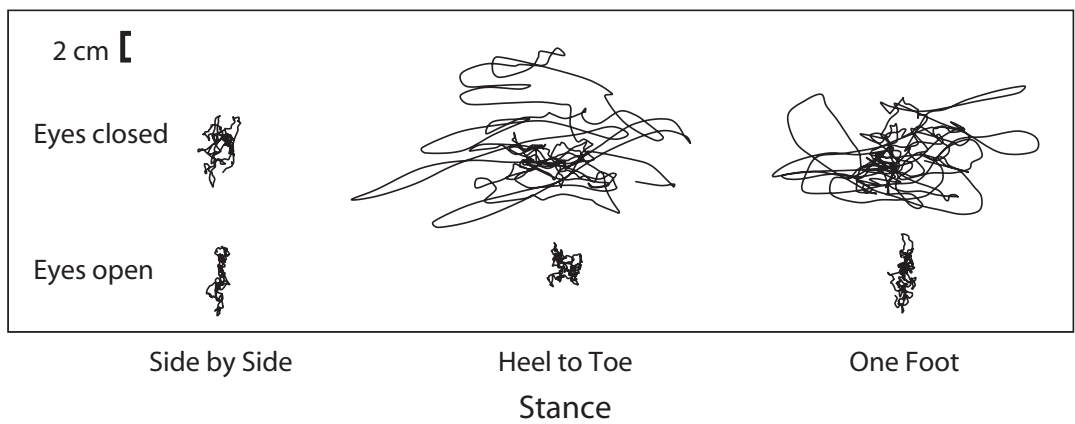

Figure 2. Raw position data from a typical subject in the real-world unrestricted field-ofview condition in Experiment 1. 


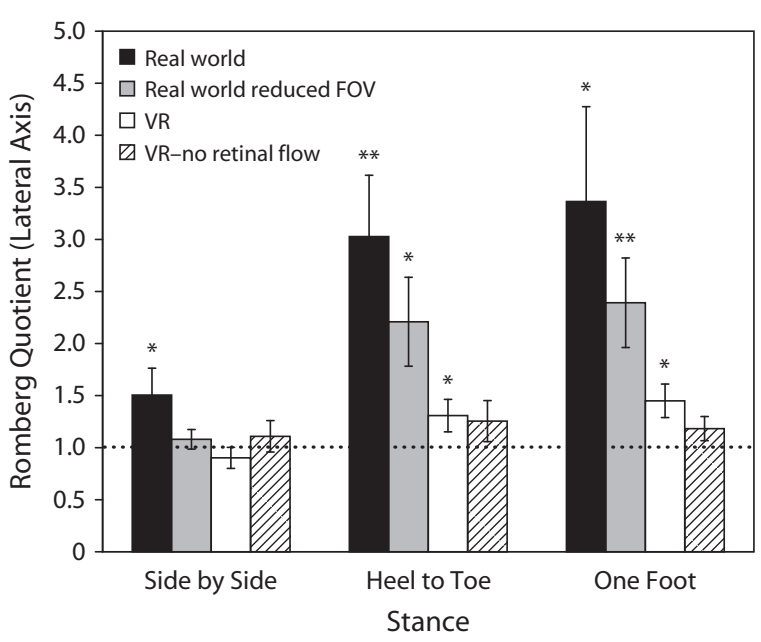

Figure 3. Romberg quotients in the lateral body axis for Experiment 1. Error bars represent $\pm 1 S E M$ and contain betweensubjects variability. For means significantly different from 1.0, ${ }^{*}$ indicates $p<.05$ and ${ }^{* *}$ indicates $p<.01$. FOV, field of view; VR, virtual reality.

side stance elicited a Romberg quotient greater than 1.0 for real-world viewing with a full FOV $(p<.05)$, but not for the other three visual conditions. Heel-to-toe and one-foot stances produced Romberg quotients significantly greater than 1.0 for real-world viewing with full and reduced FOV $(p \mathrm{~s}<.05)$, as well as for the VR display $(p \mathrm{~s}<.05)$, but not for the cyclopean VR display.

Similar analyses were conducted using the AP body sway data (presented in Figure 4). The ANOVA showed no significant main effects either for condition $[F(3,21)=$ 1.37 , n.s., $\left.\eta_{\mathrm{p}}^{2}=.16\right]$ or for stance $[F(2,14)=1.29$, n.s., $\left.\eta_{\mathrm{p}}^{2}=.16\right]$ and no significant interaction between the two $\left[F(6,42)=0.68\right.$, n.s., $\left.\eta_{\mathrm{p}}^{2}=.09\right]$. One-sample $t$ tests revealed that only the one-foot stance produced a Romberg quotient greater than 1.0, and only for real-world viewing conditions (with both full and reduced FOV).

\section{Discussion}

The primary goal of Experiment 1 was to compare the visual control of posture in real and virtual environments. The results indicate that, when a difficult stance was maintained, the virtual display provided the subjects with visual information that helped reduce body sway along the lateral body axis. However, the visual information presented through VR was less effective than the visual information under real-world viewing. In addition, the data suggest that this difference was partially, but not entirely, due to the limited FOV in the virtual display. Although the FOV limiter did reduce the stabilizing influence of vision in a real-world scene, it did not reduce it to the levels found in the VR condition. To place the FOV findings in the context of previous work, Paulus et al. (1984) reported Romberg quotients of 2.7 when subjects viewed a realworld scene with unrestricted FOV while standing on a destabilizing rubber pad. When they reduced the FOV to $30^{\circ}$, the average Romberg quotient dropped to 1.6. This reduced benefit of vision after FOV limitation is similar to our findings in Experiment 1.

The fact that vision was more stabilizing in the real environment with limited FOV than in VR is not entirely surprising, given the numerous differences that still exist between the real and the virtual scenes. Stoffregen et al. (2004) concluded that display quantization was not a major factor but that other differences still remained, such as fixed accommodation, graphics latency in response to head movement, optical distortion, and a reduced range of colors and illumination. Furthermore, distances in VR are typically underestimated when displayed via an HMD (Loomis \& Knapp, 2003; Messing \& Durgin, 2005; Thompson et al., 2004; Willemsen, Colton, Creem-Regehr, \& Thompson, 2004). Of these various factors, the tracking latency seems most likely to disrupt stability. Paulus et al. (1984) measured the effect of intermittent visual information on postural sway, using stroboscopic illumination of the visual scene. By varying the frequency of illumination between 1 and $32 \mathrm{~Hz}$, they found that frequencies higher than $16 \mathrm{~Hz}$ (i.e., more frequent than once every $62.5 \mathrm{msec}$ ) resulted in postural stability that was comparable to that with normal viewing. The latency introduced by the VR system used in Experiment $1(90 \mathrm{msec})$ lies above the threshold discovered by Paulus et al. (1984) and could be responsible for the decreased stability under virtual viewing conditions. However, tracking latency is quite different from stroboscopic illumination, since it is a constant overall lag, rather than just intermittency. Therefore, to further investigate the visual control of posture in VR, Experiment 2 manipulated the tracking latency of the VR system.

In Experiment 1, we also investigated whether a cyclopean virtual scene, containing no retinal flow, could be effectively used to control posture. Whereas other work has shown that cyclopean displays can be used to catch a ball, follow a path, and judge heading (Loomis et al., 2006;

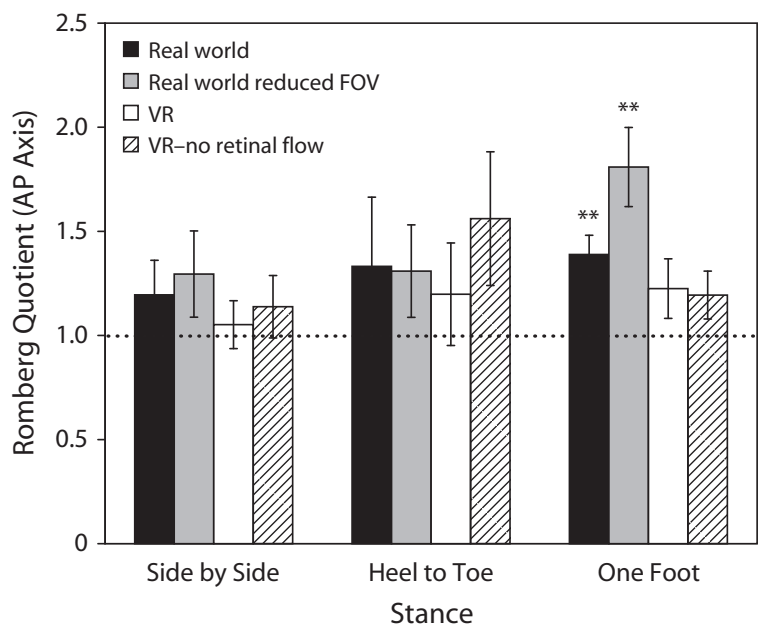

Figure 4. Romberg quotients in the anterior-posterior (AP) body axis for Experiment 1. Error bars represent $\pm 1 S E M$ and contain between-subjects variability. For means significantly different from 1.0, ${ }^{*}$ indicates $p<.05$ and ${ }^{* *}$ indicates $p<.01$. FOV, field of view; VR, virtual reality. 
Macuga et al., 2006), this stimulus was not sufficient to stabilize posture in Experiment 1. However, it was also no different from the more traditional VR display. Therefore, we believe that the failure of the cyclopean stimulus to support posture resulted from the VR system itself, which did not provide sufficient visual information to stabilize posture in most of the conditions tested. If the limitations associated with VR could be overcome, perhaps the cyclopean display could prove to be stabilizing.

The manipulation of stance also proved to be a critical one. The heel-to-toe and one-foot stances both produced a significantly greater influence of vision (seen in the larger Romberg quotients for these two stances), as compared with the side-by-side stance. Although the side-by-side stance was not sufficient to elicit visual stabilization from the virtual display, the greater inherent instability of the other two stances was critical to finding the conditions under which the virtual display was stabilizing.

\section{EXPERIMENT 2}

Experiment 2 was designed to investigate whether reducing the graphics latency in VR would increase the benefit of vision in controlling posture. This was done by comparing two different tracking systems used to measure head position. The first was the optical system used in Experiment 1, and the second was a goniometer, a multisegmented arm affixed to the top of the HMD. The goniometer system reduced the end-to-end system latency by $63 \%$ (from 90 to $33 \mathrm{msec}$; see note 1). Visual control of posture was therefore measured with real-world viewing (both with unrestricted and restricted FOV), VR with optical tracking (the same virtual display as that used in Experiment 1), and VR with goniometer tracking. Because the one-foot stance in Experiment 1 resulted in greater sensitivity in determining the stabilizing effects of vision, it was used exclusively in Experiment 2.

\section{Method}

Subjects. Eight undergraduate students ( 3 of them female) at the University of California, Santa Barbara, participated in exchange for course credit. All the subjects were verified to have at least 20-20 vision and $80 \%$ stereopsis.

Design. Experiment 2 utilized only the one-foot stance. Four visual displays were used, three of which were replications of displays used in Experiment 1 (real environment with full FOV, real environment with reduced FOV, and VR with optical tracking for Experiment 2). For the fourth display type, VR with goniometer tracking, the optical-tracking system used in Experiment 1 was replaced with a goniometer-based tracker, which reduced tracking latency by $63 \%$.

The order of visual display was counterbalanced using a balanced Latin squares design. For each of the four display types, the subjects completed one trial with eyes open and one with eyes closed, in a random order.

Stimuli and Materials. In the real-world conditions, the subjects viewed the same scene of the lab door used in Experiment 1 (Figure 1). Both virtual conditions used the same photorealistic virtual scene used in Experiment 1, containing retinal flow. The HMD and graphicsrendering equipment were identical to those in Experiment 1.

The reduced-latency virtual display used a six degree-of-freedom goniometer (Shooting Star ADL-1) attached to the top of the HMD. Subject head position was calculated on the basis of the joint angles of the goniometer, measured with rotary potentiometers, and was sent to the graphics computer over a serial line running at 57,600 baud. Using this method, latencies associated with the opticaltracking system (see note 1) were eliminated, reducing the end-toend system latency to $33 \mathrm{msec}$.

Procedure. The only notable change in the procedure was that the trial time was reduced from 60 to $20 \mathrm{sec}^{3}$ This was done because of the strenuous nature of the one-foot stance, which was used exclusively in Experiment 2.

\section{Results}

Romberg quotients for both lateral and AP body axes in Experiment 2 are presented in Figure 5. One-way repeated measures ANOVAs were conducted to assess the impact of visual display type on lateral and AP Romberg quotients. A significant main effect of display was found in the lateral body axis $\left[F(3,21)=8.52, p=.001, \eta_{\mathrm{p}}^{2}=.55\right]$. Withinsubjects contrasts showed that Romberg quotients under unrestricted real-world viewing were greater than those in all the other conditions $\left[F(1,7)=10.55, p=.014, \eta_{\mathrm{p}}^{2}=\right.$ .60]. The hypothesis that VR with goniometer tracking would result in larger Romberg quotients than would VR with optical tracking was not supported $[F(1,7)=0.15$, n.s., $\left.\eta_{\mathrm{p}}^{2}=.02\right]$. In the AP body axis, the same analysis revealed no main effect of display type $[F(3,21)=1.14$, n.s., $\left.\eta_{\mathrm{p}}^{2}=.14\right]$ and no difference between the two VR conditions $\left[F(1,7)=0.07\right.$, n.s., $\left.\eta_{\mathrm{p}}^{2}=.01\right]$.

One-sample $t$ tests were again conducted to test for Romberg quotients greater than 1.0. Results showed a stabilizing influence of all four visual displays on lateral body sway ( $p \mathrm{~s}<.05)$. In addition, visual information in real-world unrestricted viewing, as well as in both virtual displays, significantly reduced AP body sway $(p s<.05)$.

\section{Discussion}

Experiment 2 was designed to corroborate the results of Experiment 1 and, specifically, to test the hypothesis

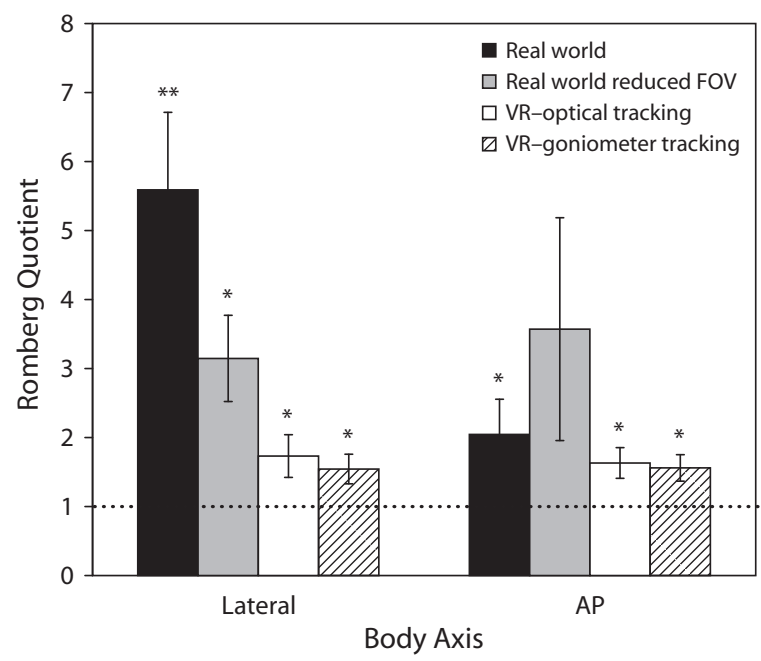

Figure 5. Romberg quotients in Experiment 2, for both lateral and anterior-posterior (AP) body axes. Error bars represent \pm 1 $S E M$ and contain between-subjects variability. For means significantly different from 1.0, ${ }^{*}$ indicates $p<.05$ and ${ }^{* *}$ indicates $p<$ .01. FOV, field of view; VR, virtual reality. 
that the reduced visual stabilization of posture in VR, as compared with real-world viewing, in Experiment 1 was partially due to the latency associated with the virtual display. Reducing the display latency in Experiment 2 did not, however, improve postural stability, and performance was no different for the two virtual displays. One potential explanation might be that the overall latency could be reduced only to $33 \mathrm{msec}$, and it is possible that further reducing the graphics latency would eventually improve performance in VR. However, the 33-msec latency was below the $63-\mathrm{msec}$ limit for normal postural stability reported by Paulus et al. (1984). Also, replicating the results from Experiment 1, the reduced effect of vision in the VR conditions could only be partially accounted for by the reduced FOV.

Overall, the results of Experiment 2 corroborated those of Experiment 1, even though the Romberg quotients were somewhat larger overall in the second experiment. This difference in Romberg quotients could be related to the shorter trial durations used in Experiment $2(20 \mathrm{sec}$, as compared with $60 \mathrm{sec}$ in Experiment 1) and, of course, the different subject population tested.

\section{GENERAL DISCUSSION}

Corroborating work by Stoffregen et al. (2004), these experiments show significant differences in postural responses to real and virtual environments. Whereas Stoffregen et al. measured postural responses to a swinging room, the present experiments extended these findings to postural control in stationary visual environments. In real environments, vision stabilized posture regardless of the subjects' stance. However, in virtual environments, only the more difficult stances (heel to toe and one foot) elicited visual stabilization of posture. Regardless of the stance used, vision was less stabilizing in the virtual then in the real environments. Still, VR has proven to be a useful tool for studying the visual control of posture. For example, Kelly et al. (2005) found that manipulating the interpretation of a 3-D virtual scene affected visually controlled posture. However, the virtual environments used in their experiments contained relative motion parallax, a visual cue available in scenes with depth information, which was not present in the present experiments.

In these experiments, we have interpreted Romberg quotients significantly greater than 1.0 as reflecting the stabilizing influence of vision due to enhanced visual feedback. However, an alternative interpretation is that posture is not stabilized by visual feedback per se but, rather, stability is improved in the service of some visual task, such as maintaining accurate fixation, which is not relevant when the eyes are closed. Indeed, research using a dual-task paradigm has shown that a secondary visual search task, which demands precise control of visual fixation and visual attention, results in improved postural stability, as compared with a simpler secondary visual task (Stoffregen, Pagulayan, Bardy, \& Hettinger, 2000). However, it seems unlikely that differences in Romberg quotients across the various conditions tested here were due to differences in visual tasks, since the task in all the environments was the same.
The present experiments show that differences between performance in real and virtual environments are not entirely due to the reduced FOV associated with virtual displays. Reducing the FOV in real-world viewing did reduce visual stabilization, but not to the levels observed in VR. Furthermore, reducing the graphics latency in the virtual display did not improve stabilization. These findings suggest that other differences in visual stimulation between real and virtual displays may be responsible for further reducing the visual stabilization of posture in the VR conditions. Some of the more relevant differences include display quantization in the HMD, optical distortion common to HMD displays, especially in the visual periphery, and the added weight of the HMD. Stoffregen et al. (2004) investigated the effects of quantization by manipulating the size of the texture elements in a virtual environment, where larger texture elements should be less subject to display quantization. However, they found no effect of this manipulation, suggesting that display quantization may not be very important. The added weight of an HMD could certainly be a source of instability, but this argument can be made for both the eyes-closed and the eyes-opened conditions. As such, the added HMD weight seems less likely to have affected the present results, which were based on the ratio of stability with eyes opened and closed. Finally, the effect of optical distortions associated with HMDs is an open question. However, Stoffregen et al. (2004) also found substantial differences between real and virtual environments using a projection-based display, which should not have had any optical distortions. This suggests that the remaining differences between real and virtual environments are not likely to be entirely due to optical distortion.

In addition to differences in visual stimulation, the extent to which subjects actually feel like they are in a real environment could also impact their performance. Despite the care taken to make the virtual scene perceptually similar to the real scene, the subjects may not have been completely immersed in the virtual world. This feeling of immersion in a virtual environment is referred to as presence. Riecke, Schulte-Pelkum, Avraamides, von der Heyde, and Bülthoff (2006) found a relationship between presence and vection, or sensed self-motion, in a visually rotating virtual scene. They manipulated presence by displaying either a photorealistic scene of a city or a scrambled version of the same scene and found that both self-reported vection and presence were greater for the normal (unscrambled) scene. However, it cannot be assumed that vection is directly related to postural sway, and further investigation is needed to assess the role of presence in postural control.

\section{AUTHOR NOTE}

Correspondence concerning this article should be addressed to J. W. Kelly, Department of Psychology, Vanderbilt University, 11121 st Avenue South, Nashville, TN 37203 (e-mail: jonathan.kelly@vanderbilt.edu).

\section{REFERENCES}

Bardy, B. G., WArren, W. H., JR., \& Kay, B. A. (1996). Motion parallax is used to control postural sway during walking. Experimental Brain Research, 111, 271-282.

Bardy, B. G., Warren, W. H., Jr., \& Kay, B. A. (1999). The role of 
central and peripheral vision in postural control during walking. Perception \& Psychophysics, 61, 1356-1368.

Begbie, G. H. (1967). Some problems of postural sway. In A. V. S. de Reuck \& J. Knight (Eds.), Myotatic, kinesthetic, and vestibular mechanisms (pp. 80-92). London: Churchill.

Brandt, T., Arnold, F., Bles, W., \& Kapteyn, T. S. (1980). The mechanism of physiological height vertigo: I. Theoretical approach and psychophysics. Acta Otolaryngologica, 89, 513-523.

Bronstein, A. M., \& BuCKWELL, D. (1997). Automatic control of postural sway by visual motion parallax. Experimental Brain Research, 113, 243-248.

Cunningham, D. W., Nusseck, H., Teufel, H., Wallraven, C., \& BüLTHOFF, H. H. (2006). A psychophysical examination of swinging rooms, cylindrical virtual reality setups, and characteristic trajectories. Proceedings of IEEE Virtual Reality, 111-118.

Diener, H. C., Dichgans, J., Bacher, M., \& GompF, B. (1984). Quantification of postural sway in normals and patients with cerebellar diseases. Electroencephalography \& Clinical Neurophysiology, 57, 134-142.

Dijkstra, T. M. H., Gielen, C. C. A. M., \& Melis, B. J. M. (1992). Postural responses to stationary and moving scenes as a function of distance to the scene. Human Movement Science, 11, 195-203.

Dijkstra, T. M. H., Schöner, G., \& Gielen, C. C. A. M. (1994). Temporal stability of the action-perception cycle for postural control in a moving visual environment. Experimental Brain Research, 97, 477-486.

EDWARDS, A. S. (1946). Body sway and vision. Journal of Experimental Psychology, 36, 526-535.

GiBson, J. J. (1950). The perception of the visual world. Boston: Houghton Mifflin.

Guerraz, M., Sakellari, V., Burchill, P., \& Bronstein, A. M. (2000). Influence of motion parallax in the control of spontaneous body sway. Experimental Brain Research, 131, 244-252.

Harwood, R. H. (2001). Visual problems and falls. Age \& Aging, 30, 13-18.

Kelly, J. W., Loomis, J. M., \& Beall, A. C. (2005). The importance of perceived relative motion in the control of posture. Experimental Brain Research, 161, 285-292.

Lasley, D. J., Hamer, R. D., Dister, R., \& Cohn, T. E. (1991). Postural stability and stereo-ambiguity in man-designed visual environments. IEEE Transactions on Biomedical Engineering, 38, 808-813.

LeE, D. N., \& Lishman, J. R. (1975). Visual proprioceptive control of stance. Journal of Human Movement Studies, 1, 87-95.

Loomis, J. M., Beall, A. C., Macuga, K. L., Kelly, J. W., \& Sмith, R. S. (2006). Visual control of action without retinal optic flow. Psychological Science, 17, 214-221.

Loomis, J. M., \& KnapP, J. M. (2003). Visual perception of egocentric distance in real and virtual environments. In L. J. Hettinger \& M. W. Haas (Eds.), Virtual and adaptive environments (pp. 21-46). Mahwah, NJ: Erlbaum.

Lu, Z. L., \& SPERLING, G. (1995). The functional architecture of human visual motion perception. Vision Research, 35, 2697-2722.

Lu, Z. L., \& SPERLING, G. (1996). Three systems for visual motion perception. Current Directions in Psychological Science, 5, 44-53.

Macuga, K. L., Loomis, J. M., Beall, A. C., \& Kelly, J. W. (2006). Perception of heading without retinal optic flow. Perception \& Psychophysics, 68, 872-878.

Messing, R., \& Durgin, F. (2005). Distance perception and the visual horizon in head-mounted displays. ACM Transactions on Applied Perception, 2, 234-250.

Mitra, S. (2003). Postural costs of suprapostural task load. Human Movement Science, 22, 253-270.

Mitra, S., \& Fraizer, E. V. (2004). Effects of explicit sway-minimization on postural-suprapostural dual-task performance. Human Movement Science, 23, 1-20.

NAKAYAMA, K., \& Loomis, J. M. (1974). Optical velocity patterns, velocity sensitive neurons, and space perception: A hypothesis. Perception, 3, 63-80

Okuzumi, H., Tanaka, A., \& Nakamura, T. (1996). Age-related changes in the magnitude of postural sway in healthy women. Journal of Human Movement Studies, 31, 249-261.
Paulus, W. M., Straube, A., \& Brandt, T. (1984). Visual stabilization of posture: Physiological stimulus characteristics and clinical aspects. Brain, 107, 1143-1163.

Paulus, W. M., Straube, A., Krafczyk, S., \& Brandt, T. (1989). Differential effects of retinal target displacement, changing size and changing disparity in the control of anterior/posterior and lateral body sway. Experimental Brain Research, 78, 243-252.

Riecke, B. E., Schulte-Pelkum, J., Avraamides, M. N., von der Heyde, M., \& Bülthoff, H. H. (2006). Cognitive factors can influence self-motion perception (vection) in virtual reality. ACM Transactions on Applied Perception, 3, 194-216.

Riley, M. A., Mitra, S., Stoffregen, T. A., \& Turvey, M. T. (1997). Influences of lean and vision on unperturbed postural sway. Motor Control, 1, 229-246.

SCHÖNER, G. (1991). Dynamic theory of perception-action patterns: The "moving room" paradigm. Biological Cybernetics, 64, 455-462.

Stoffregen, T. A., Bardy, B. G., Merhi, O., \& Oullier, O. (2004). Postural responses to two technologies for generating optical flow. Presence: Teleoperators \& Virtual Environments, 13, 601-615.

Stoffregen, T. A., Pagulayan, R. J., Bardy, B. G., \& Hettinger, L. J. (2000). Modulating postural control to facilitate visual performance. Human Movement Science, 19, 203-220.

Stoffregen, T. A., Smart, L. J., Bardy, B. G., \& Pagulayan, R. J. (1999). Postural stabilization of looking. Journal of Experimental Psychology: Human Perception \& Performance, 25, 1641-1658.

Thompson, W. B., Willemsen, P., Gooch, A. A., Creem-Regehr, S. H., Loomis, J. M., \& Beall, A. C. (2004). Does the quality of the computer graphics matter when judging distances in visually immersive environments? Presence: Teleoperators \& Virtual Environments, 13, $560-571$

van Asten, W. N. J. C., Gielen, C. C. A. M., \& van der Gon, J. J. D. (1988a). Postural adjustments induced by simulated motion of differently structured environments. Experimental Brain Research, 73, 371-383.

van Asten, W. N. J. C., Gielen, C. C. A. M., \& van der Gon, J. J. D. (1988b). Postural movements induced by rotations of visual scenes. Journal of the Optical Society of America A, 5, 1781-1789.

Van Parys, J. A. P., \& NioKikTJien, C. H. J. (1976). Romberg's sign expressed in a quotient. Agressologie, 17, 95-100.

WARREN, W. H., JR. (1998). Visually controlled locomotion: 40 years later. Ecological Psychology, 10, 177-219.

Warren, W. H.[, JR.], Kay, B. A., \& Yilmaz, E. H. (1996). Visual control of posture during walking: Functional specificity. Journal of Experimental Psychology: Human Perception \& Performance, 22, 818-838

Willemsen, P., Colton, M. B., Creem-Regehr, S. H., \& Thompson, W. B. (2004). The effects of head-mounted display mechanics on distance judgments in virtual environments. In Proceedings of the 1st Symposium on Applied Perception in Graphics and Visualization (ACM International Conference Proceeding Series, Vol. 73, pp. 35-38). New York: ACM Press.

WiTKIN, H. A., \& WAPNER, S. (1950). Visual factors in the maintenance of upright posture. American Journal of Psychology, 63, 31-50.

\section{NOTES}

1. In the virtual display used by Stoffregen et al. (2004), 1 pixel subtended $0.09^{\circ}$ of visual angle. Spatial aliasing is a direct result of pixel size.

2. Using a strobe light and an oscilloscope, the total system latency (from onset of the strobe, indicating a position change, to the subsequent movement of the display image, both measured by phototransistor probes linked to the oscilloscope) was measured to be $90 \mathrm{msec}$ on average (range, 75-100). Further measurements revealed that the graphics rendering contributed $33 \mathrm{msec}$ to this total, the remainder being due to the position-tracking system and communication between the tracking and the graphics computers (both running at $60 \mathrm{~Hz}$ ).

3. Reanalysis of the one-foot stance trials from Experiment 1, using only the first $20 \mathrm{sec}$ from each trial, did not substantially affect the pattern of results.

(Manuscript received January 17, 2007; revision accepted for publication July 16, 2007.) 